\title{
Exogenous expression of UHRF1 promotes proliferation and metastasis of breast cancer cells
}

\author{
XIN-LI LI $^{1 *}$, JIU-HONG XU ${ }^{3 *}$, JI-HUA NIE ${ }^{1}$ and SAI-JUN FAN ${ }^{2}$ \\ ${ }^{1}$ School of Public Health, and ${ }^{2}$ School of Radiation Medicine and Protection, Medical College \\ of Soochow University, Suzhou 215123, Jiangsu; ${ }^{3}$ Department of Radiotherapy, The First \\ Affiliated Hospital of Soochow University, Suzhou 215006, Jiangsu, P.R. China
}

Received January 25, 2012; Accepted April 5, 2012

DOI: $10.3892 /$ or.2012.1792

\begin{abstract}
In the present study, we investigated the role of UHRF1 (ubiquitin-like protein containing PHD and RING finger domains 1) in proliferation, invasion and migration of breast cancer cells, and the potential mechanisms were also explored. Cell proliferation was examined by 3-(4,5-Dimethylthiazol-2-yl)-2,5-diphenyl tetrazolium bromide (MTT) assay; cell cycle distribution and apoptosis were evaluated using flow cytometry; protein expression was determined by western blotting; angiogenesis of xenografts was assessed by microvessel density (MVD); cell invasion was measured using transwell chamber; cell migration was determined by wound scratching assay. Our results demonstrated that UHRF1 transfection conferred serum independence to MDA-MB-231 cells, $\mathrm{G}_{1}$ phase shortage and apoptosis suppression, accompanied with an increased expression of cyclin $\mathrm{D}_{1}$ and decreased expression of Bax. Significant pro-invasion and pro-migration activity was observed, with no obvious effect on the expression of PTEN and maspin. Co-expression of the UHRF1/PTEN or UHRF1/maspin degraded the role of UHRF1 in regulating invasion and migration. UHRF1 induced growth of MDA-MB-231 cells by promoting tumor vessel formation in vivo. In conclusion, UHRF1 promoted the proliferation of breast cancer cells by apoptosis inhibition, $\mathrm{G}_{1}$ phase shortage and promotion of tumor vessel formation, and pro-invasion and pro-migration activity was also observed by
\end{abstract}

Correspondence to: Dr Xin-Li Li, School of Public Health, Medical College of Soochow University, Suzhou 215123, Jiangsu, P.R. China

E-mail: lixinli@suda.edu.cn

Dr Sai-Jun Fan, School of Radiation Medicine and Protection, Medical College of Soochow University, Suzhou 215123, Jiangsu, P.R. China

E-mail: sjfan@suda.edu.cn

${ }^{*}$ Contributed equally

Key words: invasion, UHRF1, human, oncogene, breast cancer, cell proliferation, migration interacting with PTEN and maspin. Thus, UHRF1 may serve as a new therapy target for breast cancer.

\section{Introduction}

Breast cancer is one of the most common diseases among women world-wide, and more than $1 / 3$ breast cancer patients run high risk of recurrence and migration per year, which is the main reason that leads to poor clinical outcomes and caused death of patients. Furthermore, in China the incidence of young breast cancer patients is increasing, thus it is imperative to find more effective treatment to prevent metastasis of breast cancer patients.

Metastasis of cancer cells is a complex process involving multiple steps where cancer cells penetrate the basement membrane, intravasate into blood/lymphatic vessels, survive the journey in vasculature, extravasate into secondary sites, and adapt to new host environment (1). E-cadherin (2), matrix metalloproteinases and collagenases (3), vascular endocrine growth factor (VEGF) (4), the phosphatase and tensin homology deleted on chromosome 10 (PTEN) (5), and eukaryotic translation initiation factor 4E (eIF4E) (6) have been confirmed to play important roles in the attack and migration process of breast cancer, and there might have other genes that participate in the metastatic progression of cancer, so the exact molecular mechanisms of metastasis need to be further investigated.

UHRF1 (ubiquitin-like protein containing PHD and RING domains 1), also known as ICBP90, belonging to UHRF family, was previously cloned and isolated in a screen for proteins that bound to a CCAAT box in the promoter region of the topoisomerase II gene $(7,8)$. UHRF1 assigns to $19 \mathrm{p} 13.3$, consists of 18 exons, encodes a $95-\mathrm{kDa}$ nuclear protein of 793 amino acids, with a single open reading frame (ORF) containing an N-terminal ubiquitin-like domain, a leucine zipper motif, a central zinc finger motif of the homeodomain (PHD) finger type, a C-terminal zinc finger of the RING finger type and a ring associated (SRA) domain. It also possesses consensus sequences for an ATP/GTP binding site, a cyclin A/E-Cdk2 (cyclin dependent kinase 2) phosphorylation site, two retinoblastoma protein $(\mathrm{Rb})$-binding motifs (331LMCDE335 and 725LCCQE729) (7). Although the exact biological functions of UHRF1 are uncertain, previous studies 
in vitro showed that UHRF1 may be a growth-regulating gene because its expression is regulated during the cell cycle, and it forced post-mitotic cells to proliferate $(9,10)$. The expressions of UHRF1 mRNA and protein were low in quiescent cells and high in proliferating cells and tissues, including breast carcinomas (11), and our previous study also indicated that UHRF1 promoted growth of breast duct cancer BT-549 cells (12), and conferred radioresistance of HeLa cells (13) and breast cancer cells (14), all our results suggested its oncogene traits. However, few studies have explored whether UHRF1 could regulate proliferation in vivo, and affect invasion and migration of breast cancer cells.

In our present study, we aimed to elucidate the exact activities and the underlying mechanisms of UHRF1 in regulating proliferation, invasion and migration of breast cancer cells. Our results demonstrated that UHRF1 promoted growth of MDA-MB-231 cells in vitro, accompanying with the shortage of $\mathrm{G}_{1}$ phase, suppression of apoptosis, increased expression of cyclin $D_{1}$ and decreased expression of Bax in serumdependence experiment. In a nude murine model bearing breast cancer resulted in significant pro-growth effect, partly due to the induction of angiogenesis. Meanwhile, significant pro-invasion and pro-migration activities in dosage and time-dependent manner of UHRF1 overexpression were also observed, without affecting the proteins expression of PTEN and maspin. Co-expression of UHRF1/PTEN or UHRF1/ maspin could have a weak role in UHRF1 regulating invasion and migration. These results provide evidence that UHRF1 might be a suitable target for treatment of breast cancer.

\section{Materials and methods}

Cell culture. MDA-MB-231 and MDA-MB-453 cells were originally purchased from the American Type Culture Collection (ATCC, Manassas, VA, USA) and maintained in Dulbecco's modified Eagle's medium (DMEM, Invitrogen, Carlsbad, CA, USA), with $10 \%$ calf serum, 100 unit $/ \mathrm{ml}$ penicillin, $100 \mu \mathrm{g} / \mathrm{ml}$ streptomycin, and a mixture of non-essential amino acids (Sigma-Aldrich, St. Louis, MO, USA) at $37^{\circ} \mathrm{C}$ in an atmosphere of $5 \% \mathrm{CO}_{2}$.

Generation of UHRF1, PTEN and maspin expression plasmid. RNA isolation, cDNA synthesis, and multiplex real-time PCR were performed as previously described (14). The full-length UHRF1 cDNA was annealed and inserted into the KpnI and XhoI sites of pcDNA3 according to the manufacturer's instructions (Invitrogen). The recombinant vectors were confirmed by the digestion analysis of restriction endonuclease and all inserted sequences were verified by DNA sequencing. PTEN and maspin expression plasmids were constructed by using the same procedure described above.

Transfection. As we previously described (14), cells were plated on 6 -well plates at $2 \times 10^{5} /$ well and cultured overnight to $80 \%$ confluence, these cells were then transfected with pcDNA3 or pcDNA3-UHRF1 plasmid DNA using Lipofectamine 2000 according to the manufacturer's instruction. After incubation at $37^{\circ} \mathrm{C}$ for $5 \mathrm{~h}$, the media were replaced by fresh complete media to incubate $24 \mathrm{~h}$, then cells were trypsinized, seeded in $100-\mathrm{mm}$ dishes, and cultured further in DMEM medium with
$10 \% \mathrm{FBS}$ in the presence of G418 (300-500 $\mu \mathrm{g} / \mathrm{ml}$, Invitrogen). Thirty colonies resistant to G418 were isolated in each group and cultured further for 3 weeks, then cells from each colony were collected and submitted to RT-PCR and western blot analysis to determine the mRNA and protein expression of UHRF1, only cells resistant to G418, and with high expression of UHRF1 mRNA and protein were successfully transfected with UHRF1. Ten colonies were confirmed as stable transfected clones, and cells were multiplied and stored to conduct further study. PTEN or maspin transfection was conducted as transient transfection, namely, after being transfected with pcDNA3-PTEN, pcDNA3-maspin or pcDNA3 empty vector for $24 \mathrm{~h}$, cells were harvested to determine the mRNA and protein expression, and then submitted to further experiment.

Generation of recombinant adenoviruses. The E1-deleted adenovirus- $\beta$-gal (Ad- $\beta$-gal) was obtained from Invitrogen Therapeutics (Houston, TX, USA). A recombinant adenovirus (pAd/CMV/V5-DEST; Invitrogen) containing a DNA fragment encoding the complete amino acid sequence of human UHRF1 (Ad5-UHRF1) between the CMV promoter and the polyadenylation signal (TK $\mathrm{pA}$ ) was prepared as previously described (13).

Determination of cell proliferation in vitro. Cell proliferation was performed by 3-(4,5-Dimethylthiazol-2-yl)-2,5-diphenyl tetrazolium bromide (MTT) assay as described previously (12). Exponentially growing cells were plated in 96-well culture plates at 2,000 cells/well, after the indicated duration, $10 \mu \mathrm{l}$ of $5 \mathrm{mg} / \mathrm{ml}$ MTT ( $\mathrm{pH} 4.7$ ) was added to each well and cultured for another $4 \mathrm{~h}$, and then MTT-containing medium was replaced by $150 \mu \mathrm{l} \mathrm{DMSO} /$ well, followed by shaking for $15 \mathrm{~min}$ to dissolve formazan crystals. Absorbance at $570 \mathrm{~nm}$ was measured with a microplate reader. Cell proliferation in vitro was determined for 7 days. Cell inhibition $(\%)=(1-$ A570 of treated cells/A570 of control cells) x $100 \%$.

Assessment of cell cycle and apoptosis. Cells were cultured in DMEM containing different concentration of FCS (fetal calf serum), such as 5, 2.5, 1.25, 0.625 and $0.3125 \%$. The floated cells and the attached cells were all harvested and fixed by $5 \mathrm{ml}$ of pre-cooled $70 \%$ ethanol for at least $4 \mathrm{~h}$ at $4^{\circ} \mathrm{C}$, then washed with $1 \mathrm{X}$ PBS, resuspended in $500 \mu \mathrm{l}$ propidium iodine (PI, $10 \mu \mathrm{g} / \mathrm{ml}$ ) containing $300 \mu \mathrm{g} / \mathrm{ml}$ RNase (Sigma-Aldrich, St. Louis, MO, USA), and incubated on ice for $30 \mathrm{~min}$ away from light, filtered with a $53-\mu \mathrm{m}$ nylon mesh. Cell cycle distribution and apoptosis were calculated from 10,000 cells with ModFit LT software (Becton-Dickinson, San Jose, CA, USA) using FACSCalibur (Becton-Dickinson).

Western blot assay. The protein expression was detected by western blot assay, as previously described (14). Total whole cell lysates $(50 \mu \mathrm{g})$ was separated on sodium lauryl sulfate-polyacrylamide gel electrophoresis (SDS-PAGE) and electroblotted onto nitrocellulose membranes (Millipore, Billerica, MA, USA).

Primary antibodies included a rabbit polyclonal antiUHRF1 antiserum (1:100 dilution), anti-cyclin D1 antibody (1:500 dilution) and anti-PTEN antibody (1:500 dilution), 
A

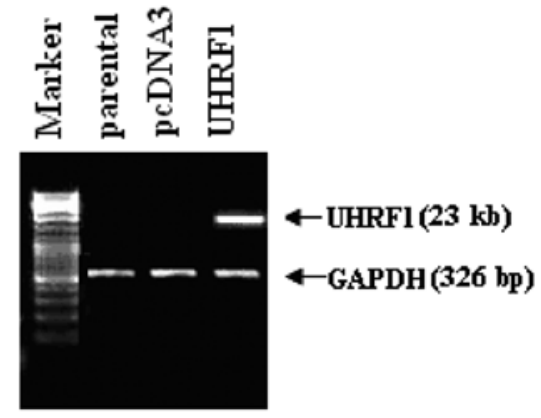

B

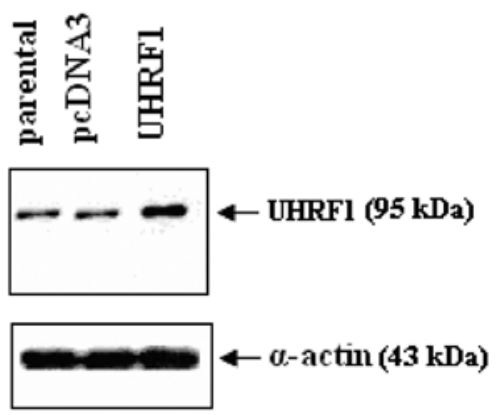

Figure 1. mRNA and protein expression of UHRF1 in breast cancer MDA-MB-231 cells after transfection. The significant expression of UHRF1 could be seen in breast cancer MDA-MB-231 cells after UHRF1 transfection as determined by RT-PCR (A) and western blot assay (B), parental cells contained an undetectable level of UHRF1 mRNA and a low level of endogenous UHRF1 protein, while no alteration was observed in the cells transfected with an 'empty' pcDNA3 control plasmid. GAPDH and $\alpha$-actin served as internal control.

which were purchased from Sigma-Aldrich. Mouse monoclonal antibodies against Bax (1:500, dilution), Bcl-2 (1:2,000 dilution) and anti-maspin antibody (1:1,000 dilution) were purchased from Sigma-Aldrich. An anti-rabbit polyclonal antibody and a goat polyclonal anti-actin antibody (1:2,000 dilutions) were obtained from Santa Cruz Biotechnology (Santa Cruz Biotechnology, Santa Cruz, CA, USA).

Preparation of tumor xenograft model. The following experimental procedures were in compliance with all regulatory guidelines and were approved by the Institutional Animal Care and Use Committee of Soochow University.

Female nu/nu BALb/c nude mice (6 to 8-week-old) were inoculated subcutaneously with breast cancer MDA-MB-231, MDA-MB-231/pcDNA3 or MDA-MB-231/UHRF1 cells $\left(\sim 5 \times 10^{6}\right.$ cells in $150 \mu \mathrm{l}$ of basic DMEM) in the right flank, each group contained 6 mice.

Determination of tumor volume. Seven days after tumor cell inoculation, tumor volumes were determined by caliper measurement of the largest (a) and perpendicular to the smallest diameter (b) every 3 days for 28 days. Data were calculated according to the formula $\mathrm{V}=1 / 6 \pi \mathrm{ab}^{2}$. The data from 3 independent experiments were combined in the analysis.

Assessment of microvessel density. The MVD (microvessel density) was quantified by the immunohistochemical ABC method as described previously (15). MVD was determined by examining vascular hot spots. Three vascular tumor areas without necrosis were selected at high magnification (x200), and the three values were averaged for each sample.

Boyden chamber assay. The invasiveness of cells was assayed by using modified Transwell Boyden chamber. Cells $\left(5 \times 10^{6}\right)$ were suspended in $50 \mu \mathrm{l}$ of serum-free medium and seeded on the upper compartment of the chamber containing PET membrane ( $8 \mu \mathrm{m}$ pore size) coated with matrigel, the lower compartment was filled with DMEM medium with 20\% FCS, the chamber was incubated at $37^{\circ} \mathrm{C}$ for $24 \mathrm{~h}$, then the upper surface of the membrane was wiped with a cotton-tip applicator to remove non-invasive cells, and the lower surface of the membrane was fixed and stained with Giemsa. Cells were quantified by counting nine high-powered fields in the center of each well using an Olympus microscope. Each measurement was performed in quadruplicate. Values shown are the mean number of cells from an equal number of view fields.

Cell migration assay. Once contact inhibition was achieved, cells were cultured in serum-free medium for $24 \mathrm{~h}, 1,000 \mu \mathrm{l}$ pipette tip was used to generate a cell-free gap of approximately $1.0 \mathrm{~mm}$ between two adjoining areas. Cells were washed twice with PBS to wipe off the loose cells, and then incubated in a $95 \%$ air $/ 5 \% \mathrm{CO}_{2}$ environment with complete medium. After the first scratch and again at 24 and $48 \mathrm{~h}$, each scratch was randomly photographed at four separate sites along the length of the scratch, starting proximally and ending distally.

Statistics. All experiments were performed at least in triplicate, results are expressed as means \pm standard deviations (SD). Statistical analysis was performed by Student's t-test. Significance was defined as $\mathrm{P}<0.05$.

\section{Results}

UHRF1 transfection increases $m R N A$ and protein expression of UHRF1. A significant expression of UHRF1 mRNA and protein could be detected after UHRF1 transfection, parental cells contained an undetectable level of UHRF1 mRNA and a low level of endogenous UHRF1 protein, while no alteration was observed in the cells transfected with an 'empty' pcDNA3 control plasmid as determined by RT-PCR (Fig. 1A) and western blot assay (Fig. 1B).

UHRF1 promotes proliferation of MDA-MB-231 cells by shortening $G_{l}$ phase and inhibiting apoptosis in vitro. Cell proliferation was determined by MTT assay for 7 days. In breast adenocarcinoma MDA-MB-231 cells (Fig. 2A), cell viability was significantly higher after being treated with UHRF1 compared with pcDNA3 transfection and parental cells $(\mathrm{P}<0.05)$, which is consistent with our previous results in breast duct cancer BT-549 cells (12).

In serum-dependence experiment, cell cycle distribution and apoptosis induction are presented in Fig. 2B, comparing with control group, overexpression of UHRF1 decreased $\mathrm{G}_{1}$ population from 68.2 to $49.9 \%$, and $\mathrm{subG}_{1}$ population (indicating apoptosis) from 11.3 to $3.9 \%$ after being treated 

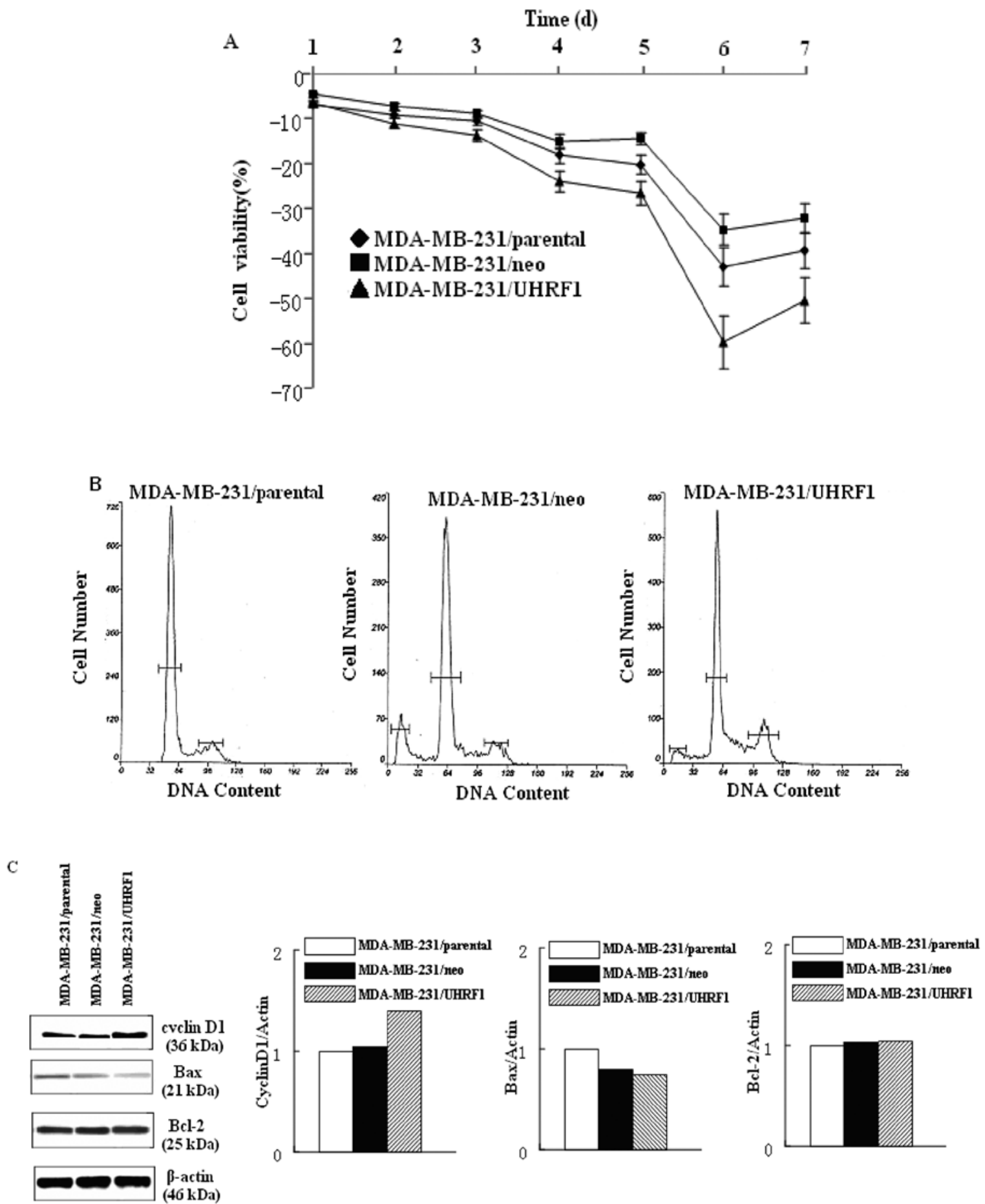

Figure 2. Effect of UHRF1 on proliferation and proteins expression relating to cell cycle and apoptosis in breast cancer MDA-MB-231 cells. (A) Cell proliferation was determined by MTT assay after UHRF1 transfection. In breast adenocarcinoma MDA-MB-231 cells, enhanced expression of UHRF1 showed significant pro-proliferation effect compared with control group and parental cells $(\mathrm{P}<0.05)$. (B) Cell cycle distribution and apoptosis was determined by flow cytometry. The enhanced expression of UHRF1 downregulated $\mathrm{G}_{1}$ and subG $\mathrm{G}_{1}$ population in MDA-MB-231 cells after cultured in complete DMEM containing $0.625 \%$ FCS. (C) Proteins expression associating with cell cycle and apoptosis were regulated by UHRF1. UHRF1 increased expression of cyclin $D_{1}$, decreased expression of Bax, but had no obvious effect on expression of Bcl-2 comparing with control group. $\beta$-actin was used as loading control. For comparison, the densitometry value for parental cells was assigned a value of 1, and values for UHRF1 transfection and empty vector transfection were normalized to 1 (foldchange vs. parental control).

with DMEM containing $0.625 \%$ FCS, and the difference was statistically significant $(\mathrm{P}<0.05)$.

Protein expression associating with apoptosis and cell cycle distribution was measured. Results reported in Fig. 2C indicate that UHRF1 transfection induced expression of cyclin $\mathrm{D}_{1}$, repressed expression of Bax, but had no effect on Bcl-2 expression compared with the control group.

UHRF1 promotes growth of MDA-MB-231 cells by inducing formation of microvessel in vivo. Comparing with the control 


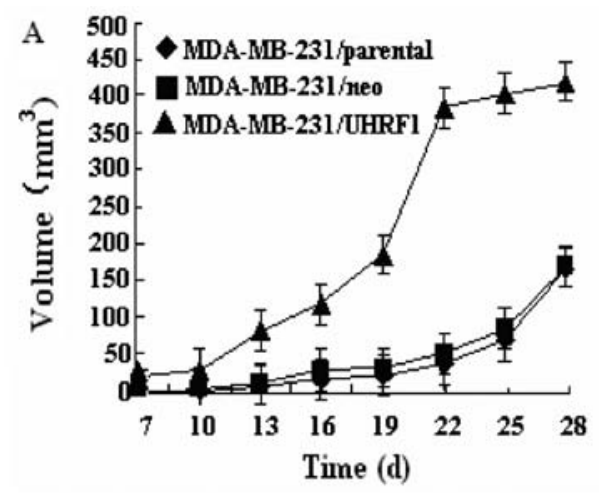

B

MDA-MB-231/parental

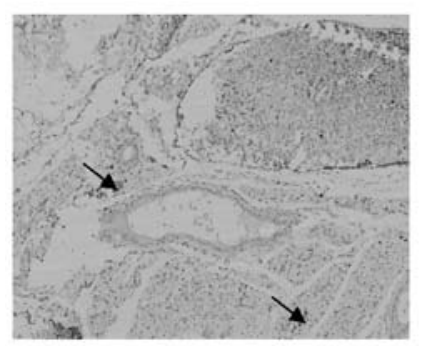

MDA-MB-231/neo

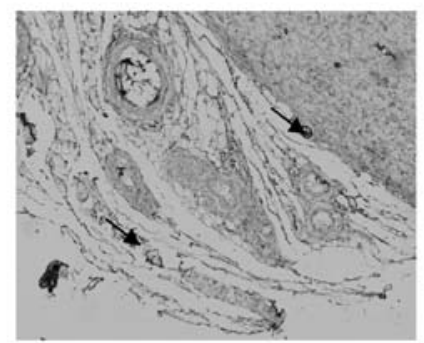

MDA-MB-231/UHRF1

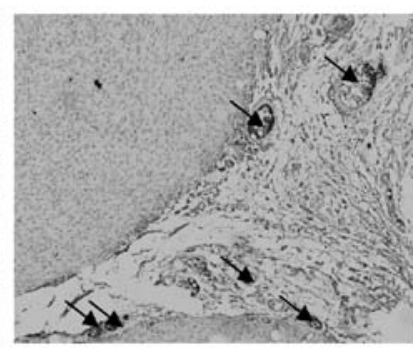

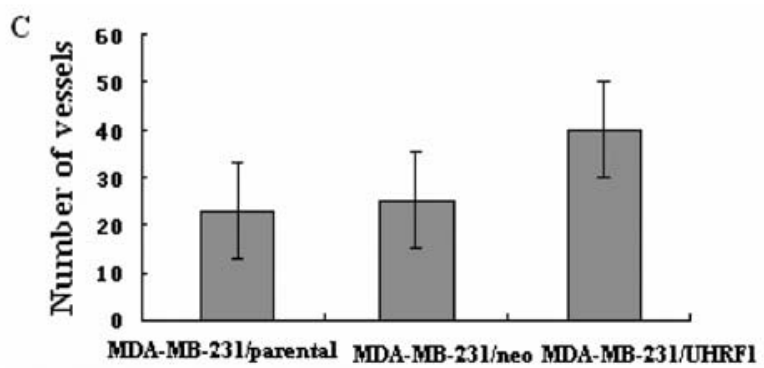

Figure 3. UHRF1 promotes tumor growth by induction of angiogenesis in vivo. (A) UHRF1 promoted tumor growth in vivo. Seven days after subcutaneous tumor inoculation, tumor volumes were measured every 3 days over the course of the experiment. Transfection with UHRF1 significantly promoted tumor growth compared with pcDNA3 transfection and parental cells. (B and C) UHRF1 induced angiogenesis in vivo. Angiogenesis in tumor tissues was evaluated by MVD assay with immunostaining against CD31. The average number of microvessels per vascular hot spot was significantly increased in UHRF1 treated tissues compared with those in the other two groups $(\mathrm{P}<0.05)$.

group, treatment with pcDNA3 did not significantly affect tumor growth $(\mathrm{P}>0.05)$, whereas UHRF1 transfection exhibited significant pro-growth as determined by volume measurements $(\mathrm{P}<0.05$, Fig. 3A). After 28 days, tumor volumes were as follows (means \pm SD): $459.34 \pm 14.800 \mathrm{~mm}^{3}$ (UHRF1, 95\% CI, 430.332-488.348), being significantly larger than the volume in the pcDNA3-treated group $\left(189.385 \pm 5.016 \mathrm{~mm}^{3}, 95 \% \mathrm{CI}\right.$, 179.554-199.216) and control group (184.357 $\pm 8.378 \mathrm{~mm}^{3}, 95 \%$ CI, 167.936-200.778).

Results of MVD assay demonstrated that the microvessels were prominent in the UHRF1 treated group but not in the control group (Fig. 3B and C). According to our data, the average number of microvessels per vascular hot spot was much smaller in the control group than in the UHRF1 treated group: $23 \pm 1.155$ (parental, 95\% CI, 21-25), 25 \pm 0.577 (pcDNA3, 95\% CI, 24-26) vs. $40 \pm 1.528$ (UHRF1, 95\% CI, 37-43, P<0.05).

UHRF1 promotes invasion of breast cancer cells. As shown in Fig. 4A, comparing with control group, there were more cells stained into blue after UHRF1 transfection, the number of invasive cells was 7-9 times more than control cells and the difference was statistically significant $(\mathrm{P}<0.01)$.

We also used the adenovirus-mediated expression of UHRF1 in breast cancer MDA-MB-231 and MDA-MB-453 cells to do the same experiment. Cells were infected with Ad5-UHRF1 or Ad5 following 8 or 24-h incubation, and then were inoculated into Boyden chamber as described above. Results shown in Fig. 4B suggest that Ad5-UHRF1 infection promoted MDA-MB-231 cell invasion in a time-dependent manner, the number of cells invaded through the membrane was 70 and 400 at 8 and $24 \mathrm{~h}$ after infection, respectively, similar result was also observed in MDA-MB-453 cells.

UHRF1 promotes migration of breast cancer cells. Results of wound scratching assay is shown in Fig. 5, at the same time point, UHRF1 transfection promoted more cells to migrate across the wound edge into the scratch area than in the control group. At $48 \mathrm{~h}$, both the MDA-MB-231/UHRF1 and control 

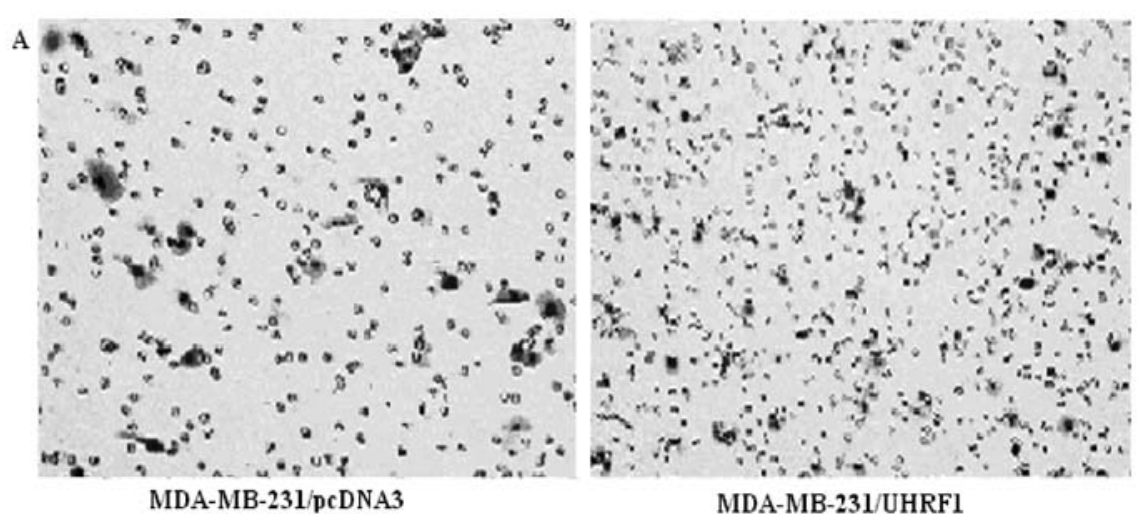

B

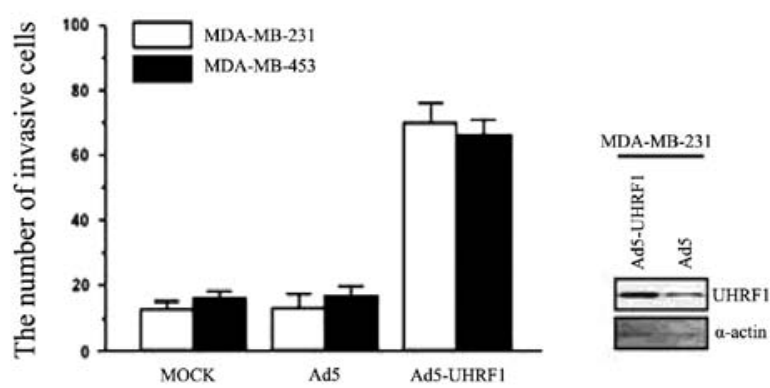

$8 h$

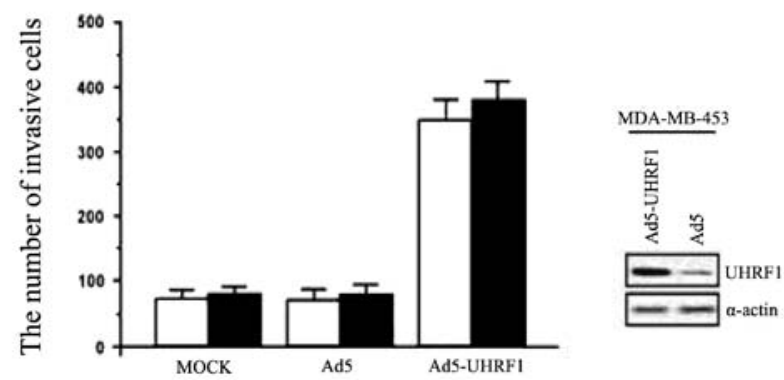

$24 \mathrm{~h}$

Figure 4. UHRF1 promotes invasion of breast cancer MDA-MB-231 cells. (A) UHRF1 transfection promoted invasion of MDA-MB-231 cells. MDA-MB-231/ UHRF1 cells and MDA-MB-231/pcDNA3 cells were diluted in $50 \mu 1$ serum-free medium at the density of $5 \times 10^{6}$ cells $/ \mathrm{ml}$, and then added to the upper chamber of the transwell chamber, the lower chamber contained $50 \mu \mathrm{l}$ complete medium supplied with $20 \% \mathrm{FBS}$. The chamber were incubated for $24 \mathrm{~h}$ in $5 \% \mathrm{CO}_{2} / 95 \%$ air at $37^{\circ} \mathrm{C}$, after being fixed and stained, invading cells were counted from four random fields of view from three independent experiments using a microscope and values shown are the mean number of cells from an equal number of view fields. (B) UHRF1 infection promoted invasion of breast caner cells in timedependent manner. Exponentially growing MDA-MB-231 and MDA-MB-453 cells were uninfected (MOCK) or infected with Ad5 or Ad5-UHRF1 at a MOI of 100 for 8 or $24 \mathrm{~h}$, then collected and inoculated into Boyden chamber as described in Materials and methods. Protein expression of UHRF1 was determined by western blot assay.

MDA-MB-231/pcDNA3 cells overlapped the wound and achieve the state of contact inhibition.

UHRF1 regulates metastasis of breast cancer cells by interacting with PTEN or maspin. Further research was performed to explore the underlying mechanisms by which UHRF1 regulated metastasis and the interactions of UHRF1 with proteins relating to metastasis. Results of protein expression in Fig. 6A demonstrated that Ad5-UHRF1 infection had no obvious effect on the proteins expression of PTEN and maspin at MOI of 50 or 100, and PTEN/UHRF1 or maspin/UHRF1 co-expression degraded the role of UHRF1 in regulating migration of breast cancer cells, as demonstrated in Fig. 6B, compared with UHRF1 infection alone, UHRF1/PTEN or
UHRF1/maspin co-expression reduced the number of cells invading the membrane from 416 to 176 and 138, respectively $(\mathrm{P}<0.05)$.

\section{Discussion}

Breast cancer is the second leading cause of death from cancer in women worldwide, although the 5-year and 10 -year survival rates of patients have increased to 70 and $50 \%$, respectively, there are still more than $30 \%$ of the patients who die of recurrence and metastasis per year.

The ability of invasion and migration for tumor is a key factor that affects the prognosis of cancer patients, research had confirmed that there are many factors affecting the above 


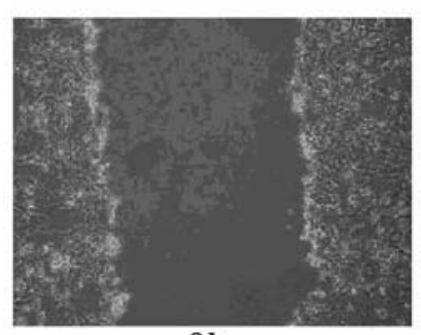

Oh

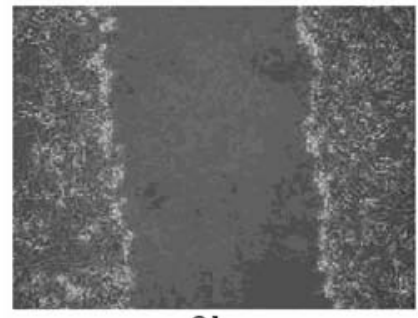

$\mathbf{0 h}$

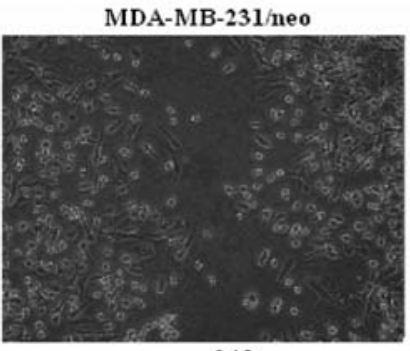

24 h

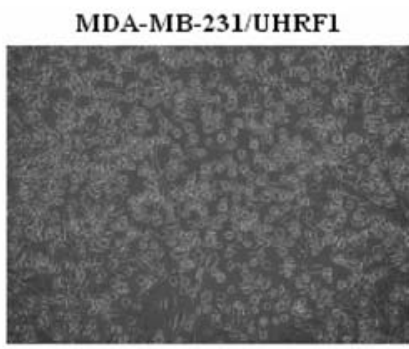

24 h

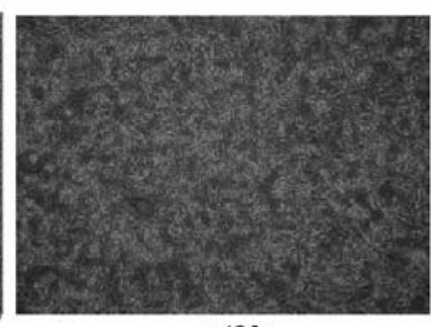

$48 h$

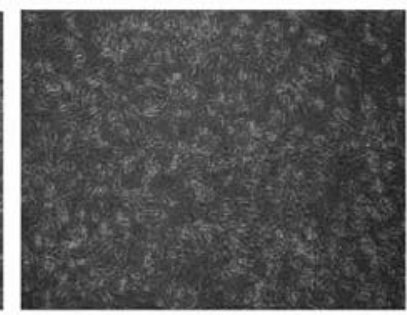

$48 \mathrm{~h}$

Figure 5. Effect of UHRF1 on migration of breast cancer MDA-MB-231 cells. Confluent MDA-MB-231/UHRF1 cells and control MDA-MB-231/neo cells were cultured in medium with free serum for $24 \mathrm{~h}$, then a cell-free zone of approximately $1 \mathrm{~mm}$ in width between two adjoining areas was created by a pipette tip scratch, cells were washed twice with PBS to wipe off the loose cells, then incubated in a $95 \%$ air $/ 5 \% \mathrm{CO}_{2}$ environment with complete medium. Each scratch was randomly photographed at four separate sites along the length of the scratch, starting proximally and ending distally. The photographs were taken on an inverted microscope immediately after the scratch and then again at 24 and $48 \mathrm{~h}$.

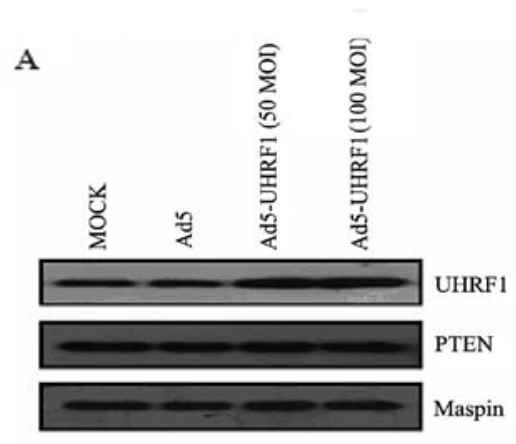

B

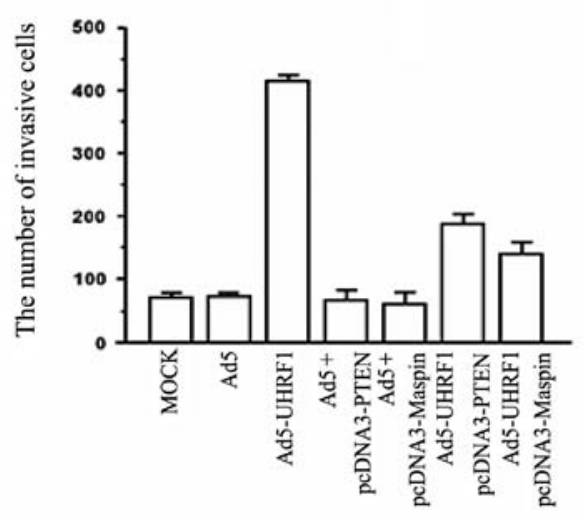

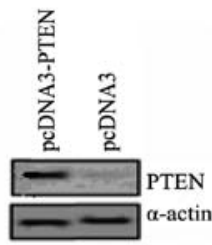

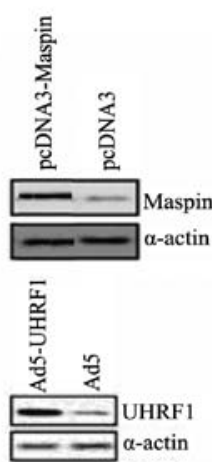

Figure 6. Interaction between UHRF1 and PTEN or maspin in MDA-MB-231 cells. (A) UHRF1 had no effect on proteins expression of PTEN and maspin. Exponentially growing MDA-MB-231 cells were mock treated or infected with Ad5 or Ad5-UHRF1 at a MOI of 50 or 100 for 48 h, then harvested and lysed, $100 \mu \mathrm{g}$ protein was electrophoresed and subjected to western blotting. $\alpha$-actin was used as a loading control. (B) Co-expression of UHRF1/PTEN or UHRF1/ maspin reduced UHRF1-mediated migration. MDA-MB-231/PTEN cells and MDA-MB-231/maspin cells were infected with Ad5 or Ad5-UHRF1 for 24 h, then cells were collected and inoculated into Boyden chamber for $24 \mathrm{~h}$, number of invasive cells was counted as described in Materials and methods. Protein expression of PTEN, maspin, and UHRF1 was determined by western blot assay.

progress, change of gene expression was one of the most important factors, so novel gene-based therapies to prevent cancer metastasis is being developed.

UHRF1 is an abnormal expression gene, we screened the radiosensitizing drug using gene-chip, in our previous studies on the gene demonstrated that UHRF1 showed significant radioresistance $(13,14)$, and to further explore its biological functions, we performed a series of experiments in breast cancer MDA-MB-231 cells to explore the effect of UHRF1 on proliferation of cells in vitro, growth of nude mice in vivo and the underlying mechanisms, the role of UHRF1 in regulating invasion and migration was also explored.

First, our results indicated that in vitro UHRF1 promoted proliferation of breast adenocarcinoma cancer MDA-MB-231 
cells (Fig. 2A), and combining with our previous results that UHRF1 also promoted growth of breast duct cancer BT-549 cells (12), we deduced that the pro-proliferation activity of UHRF1 had no relation to tissue type.

Second, UHRF1 reduced the population of $G_{1}$ and $\operatorname{subG}_{1}$ in MDA-MB-231 cells in the serum-dependence experiment (Fig. 2B), suggesting that UHRF1 endowed breast cancer cells strong serum-independence, and the pro-proliferation of UHRF1 could have been the result of $G_{1}$ cell cycle shortage and apoptosis inhibition.

Third, cyclin $\mathrm{D}_{1}$ was the critical factor regulating transition of cell cycle $\mathrm{G}_{1} / \mathrm{S}$, results (Fig. $2 \mathrm{C}$ ) revealed that UHRF1 increased the expression of cyclin $\mathrm{D}_{1}$, therefore, a potential mechanism of UHRF1 pro-tumor activity was proposed: UHRF1 upregulation results in increased cyclin $D_{1}$ activity, which results in $G_{1}$ shortage and subsequent cell proliferation promotion.

Bcl-2 family is the most prominent mediator of apoptosis induction by a large number of antitumor drugs and ionizing radiation in a variety of cell types, including cancer cells (16-18). The results of western blot analysis (Fig. 2C) revealed that UHRF1 induced downregulation of the pro-apoptosis protein Bax, which might be an important mechanism contributing to the inhibition of apoptosis, although the anti-apoptotic protein Bcl-2 was unaltered. Thus, UHRF1 upregulation results in Bax downregulation leading to inhibition of apoptosis and subsequent promotion of cell proliferation.

Fourth, UHRF1 significantly promoted tumor growth in nude mice (Fig. 3A), and the results of immunohistochemistry demonstrated that UHRF1 induced more tumor vessel formation comparing with pcDNA3 (Fig. 3B and C). Therefore, inducing tumor vessel formation might be the mechanism by which UHRF1 promoted tumor growth in vivo.

Results in Figs. 4 and 5 confirmed that both in the chemoinvasion test and wound scratching assay, MDA-MB-231/ UHRF1 cells showed stronger invasiveness compared with control MDA-MB-231/pcDNA3 cells, in other words, UHRF1 endowed cells much stronger activity of invasiveness and migration.

PTEN and maspin are two main proteins relating to cancer metastasis. Clinical research has shown that maspin played an important role in tumor growth, invasion, migration and angiogenesis $(19,20)$. Previous studies also indicated that PTEN played important roles in regulating cell adhesion, migration, differentiation, apoptosis $(4,21)$, and transfection of wild-type PTEN into highly invasive human lung cancer cells could inhibit cells invasion (22). Therefore, we deduced that regulation the expression of PTEN and maspin might be the mechanisms for UHRF1 to promote cancer cells invasion and migration, however, our results of Fig. 6A show that UHRF1 did not affect the expression of PTEN and maspin, but overexpression of PTEN and maspin could degrade the ability of UHRF1 in regulating invasion and migration for breast cancer cells (Fig. 6B), this demonstrated that PTEN and maspin might be involved in the UHRF1 regulating invasion and migration.

In summary, our results confirmed that UHRF1 promoted proliferation of breast cancer cells MDA-MB-231 by shortening the cell cycle, decreasing apoptosis accompanying with the increased expression of cyclin $D_{1}$ and decreased expression of Bax in vitro, and UHRF1 could induce tumor growth by promoting the formation of tumor vessels in vivo. UHRF1 also played a pivotal role in adjusting tumor invasion and migration by interaction with PTEN and maspin. Our results implied the possibility of UHRF1 to be applied in the treatment of breast cancer by using siRNA to downregulate its expression and to inhibit cancer cell proliferation, invasion and metastasis.

\section{Acknowledgements}

This study was supported by grants from the National Natural Science Foundation of China (no. 81001185), Universities Natural Science Foundation of Jiangsu Province (no. 10KJB310011) and the Social Development Foundation of Suzhou (no. YJS0905).

\section{References}

1. Fidler IJ: The pathogenesis of cancer metastasis: the 'seed and soil' hypothesis revisited. Nat Rev Cancer 3: 453-458, 2003.

2. Gwin K, Buell-Gutbrod R, Tretiakova M and Montag A: Epithelial-to-mesenchymal transition in metaplastic breast carcinomas with chondroid differentiation: expression of the E-cadherin repressor Snail. Appl Immunohistochem Mol Morphol 18: 526-531, 2010.

3. Daniele A, Zito AF, Giannelli G, Divella R, Asselti M, Mazzocca A, Paradiso A and Quaranta M: Expression of metalloproteinases MMP-2 and MMP-9 in sentinel lymph node and serum of patients with metastatic and non-metastatic breast cancer. Anticancer Res 30: 3521-3527, 2010.

4. Li C, Li R, Song H, Wang D, Feng T, Yu X, Zhao Y, Liu J, Yu X, Wang Y and Geng J: Significance of AEG-1 expression in correlation with VEGF, microvessel density and clinicopathological characteristics in triple-negative breast cancer. J Surg Oncol 103: 184-192, 2011

5. Esteva FJ, Guo H,Zhang S, Santa-Maria C, Stone S, Lanchbury JS, Sahin AA, Hortobagyi GN and Yu D: PTEN, PIK3CA, p-AKT, and p-p70S6K status: association with trastuzumab response and survival in patients with HER2-positive metastatic breast cancer. Am J Pathol 177: 1647-1656, 2010.

6. McClusky DR, Chu Q, Yu H, Debenedetti A, Johnson LW, Meschonat C, Turnage R, McDonald JC, Abreo F and Li BD: A prospective trial on initiation factor $4 \mathrm{E}$ (eIF4E) overexpression and cancer recurrence in node-positive breast cancer. Ann Surg 242: 584-592, 2005 .

7. Hopfner R, Mousli M, Jeltsch JM, Voulgaris A, Lutz Y, Marin C, Bellocq JP, Oudet P and Bronner C: ICBP90, a novel human CCAAT binding protein, involved in the regulation of topoisomerase IIalpha expression. Cancer Res 60: 121-128, 2000.

8. Hopfner R, Mousli M, Oudet P and Bronner C: Overexpression of ICBP90, a novel CCAAT-binding protein, overcomes cell contact inhibition by forcing topoisomerase II alpha expression. Anticancer Res 22: 3165-3170, 2002.

9. Bonapace IM, Latella L, Papait R, Nicassio F, Sacco A, Muto M, Crescenzi $M$ and Di Fiore PP: Np95 is regulated by E1A during mitotic reactivation of terminally differentiated cells and is essential for S phase entry. J Cell Biol 157: 909-914, 2002.

10. Citterio E, Papait R, Nicassio F, Vecchi M, Gomiero P, Mantovani R, Di Fiore PP and Bonapace IM: Np95 is a histonebinding protein endowed with ubiquitin ligase activity. Mol Cell Biol 24: 2526-2535, 2004.

11. Mousli M, Hopfner R, Abbady AQ, Monté D, Jeanblanc M, Oudet P, Louis B and Bronner C: ICBP90 belongs to a new family of proteins with an expression that is deregulated in cancer cells. Br J Cancer 89: 120-127, 2003.

12. Li XL, Zhu W, Zhu R and Fan SJ: Effect of human UHRF1 on growth of breast cancer BT-549 cells. Suzhou University J Med Sci 28: 345-348, 2008 (In Chinese).

13. Li XL, Meng QH and Fan SJ: Adenovirus-mediated expression of UHRF1 reduces the radiosensitivity of cervical cancer HeLa cells to gamma-irradiation. Acta Pharmacol Sin 30: 458-466, 2009.

14. Li X, Meng Q, Rosen EM and Fan S: UHRF1 confers radioresistance to human breast cancer cells. Int J Radiat Biol 87: 263-273, 2011. 
15. Yang F, Li Z, Deng H, Yang H, Yan F, Qian Z, Chen L, Wei Y and Zhao X: Efficient inhibition of ovarian cancer growth and prolonged survival by transfection with a novel pro-apoptotic gene, hPNAS-4, in a mouse model. In vivo and in vitro results. Oncology 75: 137-144, 2008.

16. Walensky LD: BCL-2 in the crosshairs: tipping the balance of life and death. Cell Death Differ 13: 1339-1350, 2006.

17. Thomadaki H and Scorilas A: BCL-2 family of apoptosis-related genes: functions and clinical implications in cancer. Crit Rev Clin Lab Sci 43: 1-7, 2006.

18. Kim R: Recent advances in understanding the cell death pathways activated by anticancer therapy. Cancer 103: 1551-1560, 2005.

19. Ravenhill L, Wagstaff L, Edwards DR, Ellis V and Bass R G-helix of maspin mediates effects on cell migration and adhesion. J Biol Chem 285: 36285-36292, 2010.
20. Sharma G, Mirza S, Parshad R, Srivastava A, Gupta SD, Pandya P and Ralhan R: Clinical significance of Maspin promoter methylation and loss of its protein expression in invasive ductal breast carcinoma: correlation with VEGF-A and MTA1 expression. Tumour Biol 32: 23-32, 2011.

21. Fabi A, Metro G, Di Benedetto A, Nisticò C, Vici P, Melucci E, Antoniani B, Perracchio L, Sperduti I, Milella M, Cognetti F and Mottolese M: Clinical significance of PTEN and p-Akt co-expression in HER2-positive metastatic breast cancer patients treated with trastuzumab-based therapies. Oncology 78: 141-149, 2010.

22. Hong TM, Yang PC, Peck K, Chen JJ, Yang SC, Chen YC and Wu CW: Profiling the downstream genes of tumor suppressor PTEN in lung cancer cells by complementary DNA microarray. AM J Respir Cell Mol Biol 23: 355-363, 2000. 